09

\title{
Лазерно-интерференционная система детектирования гравитационных волн
}

\author{
() Г.И. Долгих
}

Тихоокеанский океанологический институт им. В.И. Ильичёва ДВО РАН, Владивосток, Россия

E-mail: dolgikh@poi.dvo.ru

Поступило в Редакцию 14 марта 2018 г.

Обсуждаются новые принципы регистрации гравитационных волн на основе применения разнесенных на большие расстояния лазерных деформографов маятникового типа. Располагая три лазерных деформографа маятникового типа с длинами измерительных плеч $1000 \mathrm{~m}$ на сторонах равностороннего треугольника, равных $316 \mathrm{~km}$, можно обеспечить чувствительность данной системы в двадцать третьем знаке в частотном диапазоне от 3 до $950 \mathrm{~Hz}$ при регистрации сигналов внеземного происхождения, распространяющихся со скоростями, равными скорости света.

DOI: $10.21883 /$ PJTF.2018.20.46805.17288

В [1] сообщается о первой регистрации гравитационных волн, возникших в результате взаимодействия черных дыр, посредством использования специально созданной системы разнесенных на расстояние $3003 \mathrm{~km}$ лазерных интерферометров равноплечего типа [2]. В работе была реализована гениальная идея применения интерферометров Майкельсона для регистрации гравитационных волн, выдвинутая еще 1962 г. [3]. При создании интерферометров использовалась идея о том, что если гравитационная волна распространяется вдоль оси $x$ и если в плоскости $y z$ разместить свободные точечные массы, то из-за конечной разности расстояний между массами можно наблюдать колебания одной массы относительно другой. Если пара масс расположена вдоль оси $y$, то величина амплитуды колебаний $\Delta l$ в направлении $y$ равна

$$
\Delta l=\frac{1}{2} h l
$$

где $l$ - расстояние между массами, $h$ - амплитуда возмущения метрики, вызванного гравитационной волной. Если пара масс располо- 
жена вдоль оси $z$, то колебания будут в противофазе по отношению к колебаниям первой пары [4]. В качестве пробных масс в интерферометре использованы далеко разнесенные, свободно подвешенные массивные зеркала. Ожидается, что в ближайшее время после серии улучшений пороговая чувствительность лазерных интерферометров, аналогичных используемым в проекте LIGO, достигнет значений, лучших чем $4 \cdot 10^{-23}$, в диапазоне частот от 30 до $500 \mathrm{~Hz}$ [5]. В настоящей работе рассмотрен другой принцип построения измерительных систем, направленных на регистрацию гравитационных волн, которые могут иметь лучшие характеристики, чем отмеченные выше.

В качестве основного узла измерительной системы предлагается использовать лазерный деформограф маятникового типа, конструктивные особенности которого описаны в [6], первый опыт применения действующего деформографа маятникового типа приведен в [7]. При создании нашей измерительной системы используем отличительные особенности амплитудно-частотной характеристики лазерного деформографа маятникового типа (по сравнению с амплитудно-частотной характеристикой лазерного деформографа классического типа), позволяющие проводить абсолютные измерения смещений в высокочастотной области спектра. Так, при создании лазерного деформографа с измерительным плечом длиной $1000 \mathrm{~m}$ и маятниковой системы с резонансной частотой порядка $0.3 \mathrm{~Hz}$ можно уже говорить об измерении абсолютных смещений начиная с $3 \mathrm{~Hz}$ до высоких частот, определяемых быстродействием электронно-оптических элементов деформографов. При этом мы повышаем частоту, начиная с которой можно говорить об абсолютных измерениях, уменьшая длину измерительного плеча, и уменьшаем ее, понижая резонансную частоту маятниковой системы. В качестве пробной массы, на которую действует гравитационная волна, будем рассматривать гранитный блок, на котором смонтирована оптическая скамья основной части лазерного деформографа маятникового типа. При жестком соединении оптической скамьи с гранитным блоком можно рассматривать данный блок в качестве пробной массы (относительно длины гравитационной волны, имеющей скорость порядка $300000 \mathrm{~km} / \mathrm{s}$ ). Таким образом, можно утверждать, что в лазерном деформографе маятникового типа используется одна пробная масса. Располагая два лазерных деформографа маятникового типа на большом удалении друг от друга, можно измерить изменение расстояния между двумя гранитными блоками (пробными массами) каждого лазерного

Письма в ЖТФ, 2018, том 44, вып. 20 
деформографа, вызванное различными процессами, в том числе гравитационными волнами. Для правильного решения поставленной задачи (регистрации гравитационных волн) необходимо использовать три разнесенных на большие расстояния лазерных деформографа маятникового типа с образованием равностороннего треугольника, но расположенные в пределах одной длины гравитационной волны. В каждой точке необходимо применение системы точного времени, обеспечивающей точность $1 \mu \mathrm{s}$, что в современных условиях не является сложной задачей.

Определим минимальные расстояния, на которые мы должны разнести лазерные деформографы, чтобы обеспечить чувствительность системы в двадцать третьем знаке для регистрации гравитационной волны, т. е.

$$
\varepsilon=\frac{\Delta L}{L}=1 \cdot 10^{-23},
$$

где $L-$ расстояние между лазерными деформографами. В каждом лазерном деформографе предполагается использование частотностабилизированных лазеров, обеспечивающих кратковременную стабильность частоты в четырнадцатом знаке:

$$
\varepsilon_{1}=\frac{\Delta v}{v}=1 \cdot 10^{-14}
$$

где $v-$ частота лазера. Для конкретного лазерного деформографа это обеспечивает чувствительность

$$
\varepsilon_{2}=\frac{\Delta l}{l}=1 \cdot 10^{-14}
$$

где $l$ - длина плеча лазерного деформографа, т.е. в нашем случае $1000 \mathrm{~m}$. Расчеты проводим для граничной частоты $3 \mathrm{~Hz}$. Если принять во внимание, что скорость гравитационного возмущения составляет $300000 \mathrm{~km} / \mathrm{s}$, то при частоте, например, $3 \mathrm{~Hz}$ длина данной волны будет равна $100000 \mathrm{~km}$. Применение часов точного времени, синхронизирующих измерительные комплексы, разнесенные на расстояние $L$, с точностью $1 \mu \mathrm{s}$, позволит отслеживать изменение фазы гравитационной волны с точностью $\left(10^{8} / L\right) \cdot 10^{-6}$. Принимая во внимание последнее выражение и выражение для $\varepsilon_{2}$, можно записать уравнение для опре-

Письма в ЖТФ, 2018, том 44, вып. 20 
деления $L$ с целью достижения заданной чувствительности в двадцать третьем знаке

$$
\frac{\varepsilon_{2}}{L} \frac{10^{8}}{L} \cdot 10^{-6}=1 \cdot 10^{-23}
$$

Из этого уравнения следует, что $L \approx 316000 \mathrm{~m}$. При уменьшении частоты регистрируемых колебаний чувствительность падает, а при увеличении указанной частоты возрастает. Рассчитаем предельную частоту, для которой длина гравитационной волны не превышает максимального расстояния между опорными точками. Для нашего случая она примерно равна $950 \mathrm{~Hz}$. Таким образом, рабочий частотный диапазон данной системы маятниковых лазерных деформографов, обеспечивающих чувствительность $10^{-23}$, находится в пределах от 3 до $950 \mathrm{~Hz}$, что лучше чувствительности систем проекта LIGO.

Отдельно каждый лазерный деформограф маятникового типа не годится для регистрации гравитационных волн, так как его чувствительность ограничена стабильностью частоты лазера (четырнадцатый порядок). Если считать достоверной информацию о регистрации гравитационных волн установками LIGO (особенно с учетом того, что основным авторам присудили в 2017г. Нобелевскую премию), то лазерным деформографам маятникового типа не хватает восьмидевяти порядков. Весь смысл в применении системы пространственно разнесенных лазерных деформографов маятникового типа и методики проведения эксперимента. Главное условие: если мы считаем, что гравитационные волны есть и их скорость равна скорости света, то пространственно разнесенные приборы должны измерять сигнал, находящийся в пределах $2 \pi$ (или в терминах длины волны расстояние между двумя деформографами должно быть не больше длины гравитационной волны). При таком условии будем измерять смещения на деформографах, находящихся в пределах одной длины волны. Мы считаем, что в каждой установке уровень всех шумов меньше шумов, вызванных нестабильностью частоты рабочего лазера. Изменение фазы волны отслеживается с применением систем точного времени, использующихся в установках и синхронизированных между собой. Применение систем точного времени, обеспечивающих измерение временно́го интервала с точностью $1 \mu \mathrm{s}$, позволяет на несколько порядков поднять чувствительность системы, состоящей из двух пространственно разнесенных лазерных деформографов маятникового типа. При регистрации гравитационных волн, которые,

4 Письма в ЖТФ, 2018, том 44, вып. 20 
допустим, имеют частоту $160 \mathrm{~Hz}$, мы будем двумя лазерными деформографами прописывать амплитудно-частотные вариации гравитационных сигналов. Невозможно придумать сигналы в этом частотном диапазоне (сейсмические и т.п.), которые имели бы длины волн, позволяющие проводить их измерения в одной фазе. Дальнейший анализ экспериментальных данных, полученных на системе из трех лазерных деформографов, находящихся на сторонах равностороннего треугольника, должен проводиться с учетом следующих условий и по следующей схеме: 1) создаем модели различных гравитационных сигналов и модели их проявления на записях системы из трех пространственно разнесенных маятниковых лазерных деформографов с учетом их поляризационных свойств; 2) считаем, что скорость распространения данных сигналов равна около $300000 \mathrm{~km} / \mathrm{s}$; 3) полагаем, что шумовые сигналы земного происхождения в рассматриваемом диапазоне частот $(3-950 \mathrm{~Hz})$ для каждой установки свои и не связаны друг с другом, хотя это и не обязательное условие; 4) в качестве главного принимаем условие, заключающееся в том, что скорость распространения данных шумовых сигналов значительно меньше скорости распространения гравитационных волн; 5) проводим свертку созданных моделей гравитационных сигналов с полученными записями системы, состоящей из трех пространственно разнесенных маятниковых лазерных деформографов, т.е. „прогоняем“ модели по записи системы; 6) при совпадении модели с сигналом, даже сильно зашумленным, свертка даст резкое увеличение амплитуды на записи в месте их совпадения, что и будет доказательством регистрации гравитационного сигнала модельного вида. Результат свертки может поднять чувствительность системы еще на шесть-семь порядков. При этом следует учесть, что таких записей будет три (по сторонам треугольника), а с учетом поляризационных свойств гравитационной волны мы точно определим направление на источник данного сигнала. При желании на Земле можно построить несколько таких измерительных систем по аналогии с проектом LIGO.

Кратко остановимся на шумах, уровень которых должен быть ниже определенной величины. Этот уровень для каждой установки определяется стабильностью частоты рабочего лазера, равной $10^{-14}$. Основной источник шумов обусловлен вариациями температуры, давления, влажности, шумами фотоэлектронной аппаратуры, сейсмическими шумами природного и техногенного происхождения. Оценим порог чувствитель-

Письма в ЖТФ, 2018, том 44, вып. 20 
ности, определяемый дробовым шумом фотоприемника. Предельная чувствительность к абсолютным смещениям зеркал в интерферометре Майкельсона, ограниченная только дробовым шумом фотоприемника, определяется выражением

$$
\Delta l_{\min }=\frac{1}{4 \pi}\left\{\frac{\lambda \hbar c \Delta f}{q P_{0}}\right\}^{1 / 2}
$$

где $\lambda$ - длина волны, $P_{0}-$ мощность лазерного излучения, $c-$ скорость света, $\Delta f$ - полоса принимаемых частот, $q-$ квантовый выход фотоприемника. Полагая $\lambda=0.63 \cdot 10^{-6} \mathrm{~m}$ (гелий-неоновый лазер), $\quad P_{0}=0.01 \mathrm{~W}, q=0.25, c=3 \cdot 10^{8} \mathrm{~m} / \mathrm{s}, \hbar=6.626 \cdot 10^{-34} \mathrm{~J} \cdot \mathrm{s}$, $\Delta f \approx 10^{3}$, имеем $\Delta l_{\min }=1.78 \cdot 10^{-14} \mathrm{~m}$, что не оказывает влияния на точность измерения. При увеличении мощности лазера дробовый шум фотоприемника падает, что также используется в проектах типа LIGO. Так же как и в интерферометрах LIGO, луч лазера распространяется в трубах с глубоким вакуумом (лучше изготовленных из элинвара). Маятниковые лазерные деформографы должны быть размещены в подземных гидротермоизолированных помещениях с постоянством температуры, давления и влажности. Для ликвидации возможных вариаций метеорологических параметров, влияющих на интерферометрическую часть, каждый ее элемент должен быть изготовлен по компенсационной схеме, частично описанной в [8], в которой производится тщательный подбор компенсационных материалов и при которой практически любые изменения метеорологических параметров не оказывают влияния на точность измерения смещений. Влияние сейсмических шумов природного и техногенного происхождения на измеряемые параметры сводится к нулю при соблюдении условий и схемы, описанных выше в пунктах $1-6$.

\section{Список литературы}

[1] Abbott B.P. et al. (LIGO Scientific Collaboration and Virgo Collaboration) // Phys. Rev. Lett. 2016. V. 116. N 6. P. 061102.

[2] Abbott B.P., Abbott R., Adhikari R. et al. // Rep. Prog. Phys. 2009. V. 72 . N 7. P. 076901.

[3] Герценштейн М.Е., Пустовойт В.И. // ЖЭТФ. 1962. Т. 43. В. 2. С. 605-607.

4* Письма в ЖТФ, 2018, том 44, вып. 20 
[4] Abramovici A., Althouse W.E., Drever R.W.P., Gursel Y., Kawamura S., Raab F.J., Shoemaker D., Sievers L., Spero R.E., Thorne K.S., Vogt R.E., Weiss R., Whitcomb S.E., Zucker M.E. // Science. 1992. V. 256. N 5055. P. $325-333$.

[5] Пустовойт В.И. // УФН. 2016. Т. 186. № 10. С. 1133-1152.

[6] Долгих Г.И. // Письма в ЖТФ. 2011. Т. 37. В. 5. С. 24-30.

[7] Dolgikh G.I. // Earthq. Sci. 2015. V. 28. N 4. P. 311-317.

[8] Dolgikh G.I., Dolgikh S.G., Kovalyov S.N., Chupin V.A., Shvets V.A., Yakovenko S.V. // J. Marine Sci.Technol. 2009. V. 14. N 4. P. 436-442. 